\title{
Radiation output system for planar gyrotron
}

\author{
Dmitry Sobolev ${ }^{1, *}$, Vladislav Zaslavsky ${ }^{1}$, Naum Ginzburg $^{1}$, and Andrey Kuftin ${ }^{1}$ \\ ${ }^{1}$ Institute of Applied Physics of the Russian Academy of Sciences, 46 Ulyanov st., 603950 Nizhny \\ Novgorod, Russia
}

\begin{abstract}
Gyrotrons with planar resonator cavity offer some advantages over conventional cylindrical ones for submillimetre wavelengths. The radiation in the planar gyrotron should be converted from the mixture of several high modes of the rectangular waveguide into the Gaussian beam with high efficiency. For the experimental $145 \mathrm{GHz}$ gyrotron, a twochannel output is considered. Both channels consist of waveguide converter and two convex mirrors and direct the radiation through the single output window. Calculated total efficiency of the system exceeds $98 \%$.
\end{abstract}

\section{Introduction}

At present, there is great interest in the development of high-power terahertz radiation sources for numerous scientific and technological applications, including spectroscopy, plasma diagnostics, communications, medicine, etc. Gyrotrons are most promising among the existing subterahertz and terahertz radiation sources. A significant progress in development of subterahertz gyrotrons is achieved in the last few years. At a frequency of 1 $\mathrm{THz}$, the radiation power of conventional gyrotrons rose to several kilowatts. To get high power at these frequencies in small transverse size of the cavity, a traditional gyrotron with cylindrical cavity requires high electron beam compression and high current density in interaction space. These problems could be solved by developing modified schemes of gyrotrons with different configurations of the interaction space and directions of energy extraction. For example, a novel concept of a high-power wideband gyrotron based on planar geometry of the interaction space with sheet electron beam and transverse (with respect to the electron translation velocity) electromagnetic energy extraction is suggested in [1]. An advantage of the proposed planar scheme compared with the conventional cylindrical one is the possibility to ensure effective mode selection along the open transverse coordinate in combination with radiation out-coupling, which leads to a significant reduction of ohmic losses [2]. The radiation at each side of the cavity can be represented as the a mixture of several modes of rectangular waveguide with high index at one coordinate and several low indexes at another coordinate. This radiation must be directed into the output windows, preferrably at the form of the wave beam with high Gaussian content and low losses on the way. A unique component is required to ensure efficient conversion between these field distributions, and its shape can be synthesized using the method developed by authors [3].

*Corresponding author: nrtnm@mail.ru 


\section{Radiation output layout}

The radiation is extracted from the gyrotron cavity in directions orthogonal to the magnet axis. There are two flat mirrors tilted by $45^{\circ}$ to the magnet axis, one on each side, that guide the radiation forward to avoid interference with magnet inner wall (Fig. 1). The radiation propagates forward in two channels that can be either mixed later or left separate. For the experimental gyrotron, the latter layout is preferable, because it is yet unknown if production tolerances lead to significant difference between power in two channels. That difference could cause the distortion of the output field structure if two channels are mixed. Another advantage of this layout is complete separation of electron path and radiation path after the cavity, which significantly eases the collector design.
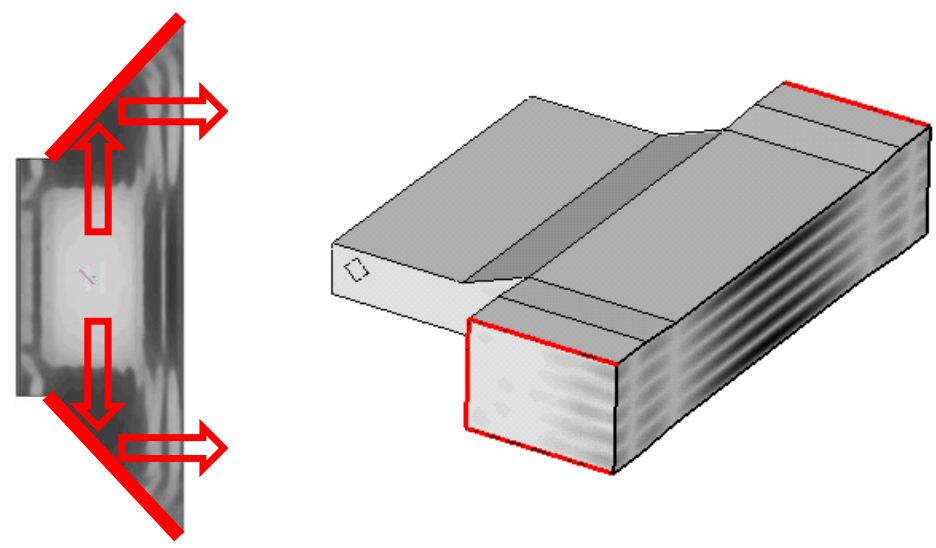

Fig. 1. The cavity and electric field structure of the experimental planar gyrotron.

The experimental gyrotron developed to prove the planar design concept has the cavity size $4 \mathrm{~cm}$ by $2 \mathrm{~cm}$ by $1.15 \mathrm{~cm}$ and operation frequency $144.8 \mathrm{GHz}$. Each output channel has therefore cross-section $2 \mathrm{~cm}$ by $1.15 \mathrm{~cm}$. According to numerical simulations, the electromagnetic field at the start of each output channel can be represented as a mixture of several first $\mathrm{TE}_{N, 11}$ modes of the rectangular waveguide, where $N=0 . .4$. The content and phase of individual modes may slightly change depending on generation parameters, but either way the ratio of transverse wave numbers in orthogonal directions is at least 5, and therefore the most part of the radiation in output channels propagates at a small angle to the short wall plane and at a big angle to the long wall plane. This make the conversion to the modes with different second index much easier than to modes with different first index. The initial mode mixture in each channel is converted into the tilted Gaussian wave beam by its own synthesized waveguide converter, which will be discussed later. Two wave beams are guided by two pairs of convex mirrors. The beams are crossing at the small angle when they pass through the single output window into one or two calorimetric loads. Two different loads may be useful to analyse the asymmetry of the cavity. If waveguide converters produce high quality beams, then simple elliptic shape of the mirrors is sufficient to transmit all power to the loads.

\section{Synthesized waveguide converter}

The mixture of high modes is converted into Gaussian wave beam by a specially shaped irregular waveguide, which surface was synthesized using iterative numerical optimization 
method developed earlier by authors. The method uses only two field calculations per iteration and usually needs several tens of iterations to converge. At the beginning of every iteration step the system (Fig. 2) consists of the given input cross-section $S_{1}$, given output cross-section $S_{2}$ and initial conducting wall surface $S_{0}$. If we consider only one orientation, then conversion efficiency can be is increased after iteration by calculating two field distributions inside the waveguide. The first one is calculated using the given $\mathbf{E}_{1}$ and $\mathbf{H}_{1}$ fields at the input as a boundary condition, the second one by using the spatially reversed desired $\mathbf{E}_{-2}$ and $\mathbf{H}_{-2}$ fields at the output.

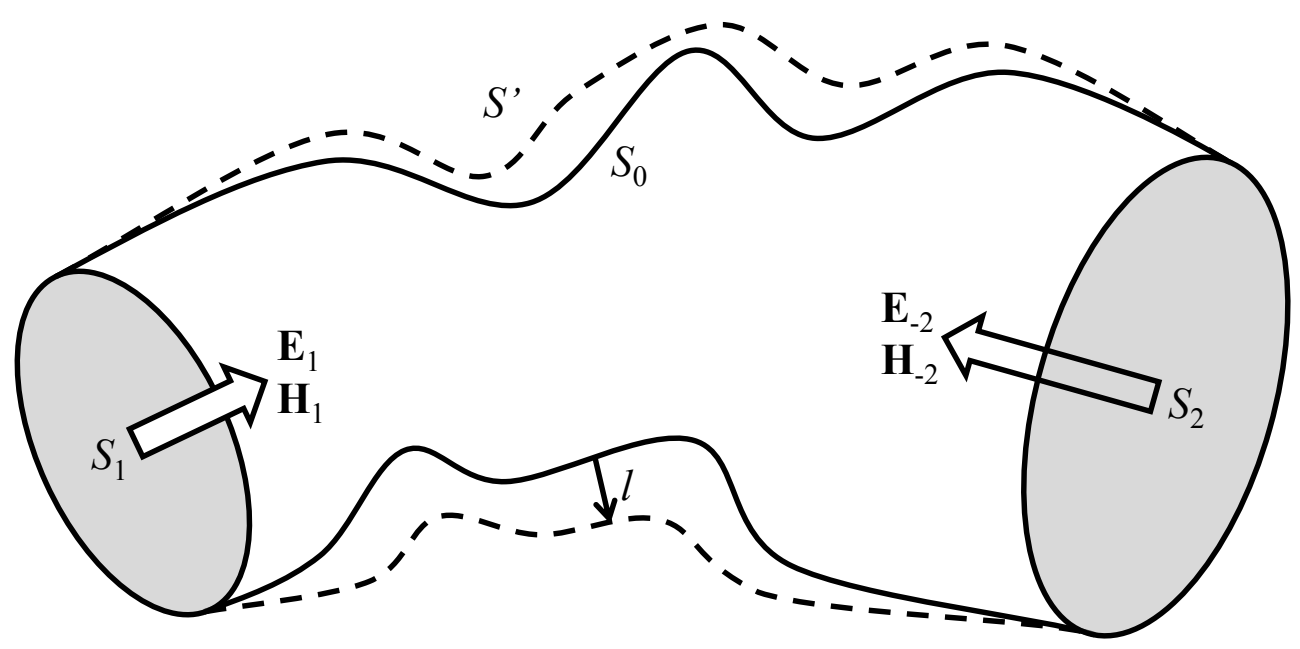

Fig. 2. Notations used in iterative synthesis method.

Small and smooth deformation $l$ orthogonal to waveguide wall affects the efficiency (mutual power) $P$ according to formulas

$$
\Delta P=\int_{S_{0}} l F d S, \quad F=-i k\left(\mathbf{H}_{\tau 1} \mathbf{H}_{\tau-2}+\mathbf{E}_{n 1} \mathbf{E}_{n-2}\right)
$$

where $\tau$ and $n$ means tangential and normal field components, respectively. We can minimize the average square of deformation (i.e. norm) using real and imaginary parts of (1) as equality constraints with given $\Delta P$, and in this simple case the deformation takes the form

$$
l=\alpha \operatorname{Re} F+\beta \operatorname{Im} F
$$

where constants $\alpha$ and $\beta$ are expressed by $\Delta P$ and surface integrals of function $F$ [3].

The converter has a length of $14 \mathrm{~cm}$ and consists of two parts (Fig. 3). The first $7 \mathrm{~cm}$ is the shaped taper with the small wall size increasing from $1.15 \mathrm{~cm}$ to $1.5 \mathrm{~cm}$ and conserving the initial mode content, which helps to tilt the output beam at the required direction $\left(50^{\circ}\right.$ to the waveguide axis). The second part converts $\mathrm{TE}_{N, 11}$ mode mixture into special $\mathrm{TE}_{N, M}$ mode mixture (most part of the total power is in the modes with $9 \leq M \leq 13$ ) which has over $99 \%$ coupling with the inclined Gaussian beam. If we reverse the principal ray of the output Gaussian wave beam back into the second part of the converter, it is reflected only 5 times before reaching the input. To confirm the efficiency of the converter, additional calculations were performed, using both time-domain (FDTD) methods and frequencydomain surface integral equation methods (MoM-MLFMA). The total calculated efficiency of the output system is over $98 \%$. 

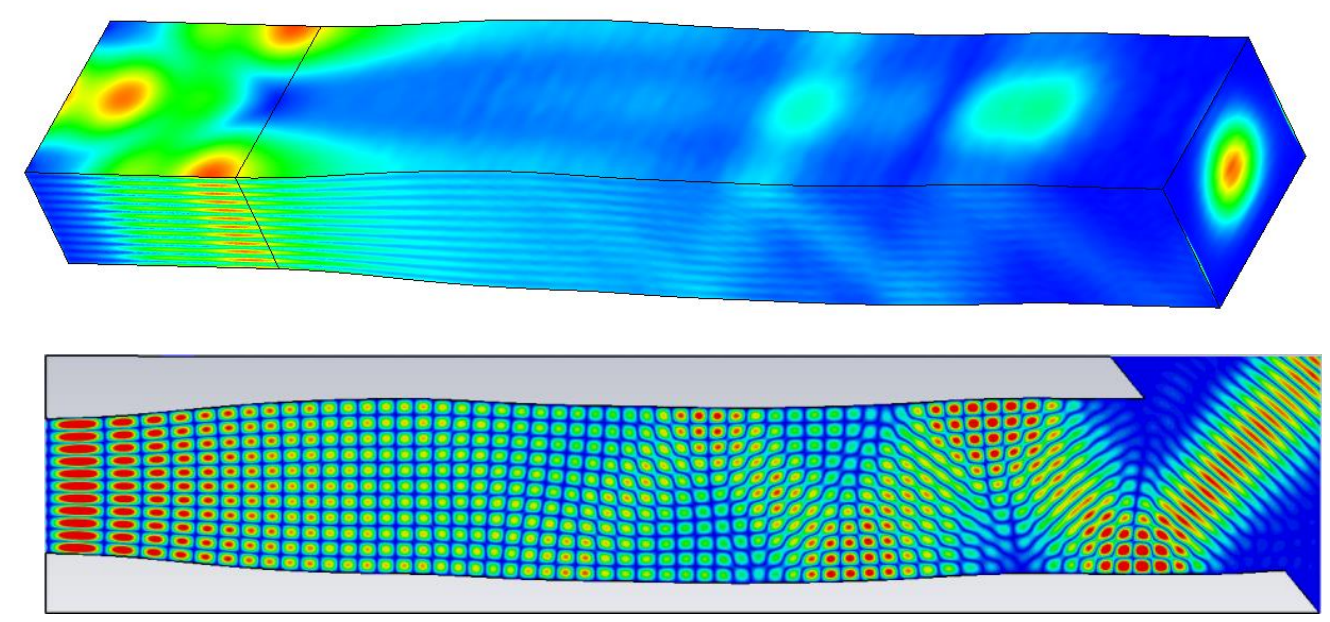

Fig. 3. Absolute value of the electric field on the walls of the synthesized waveguide converter (top) and snapshot of the electric field in its cross-section (bottom).

\section{Conclusion}

The output system developed for the experimental planar gyrotron allows the extraction of the output power with high efficiency and with ability to monitor the difference between signals from different sides of the cavity.

This work was funded by Russian Science Foundation grant 18-19-00704.

\section{References}

1. N.S. Ginzburg, I.V. Zotova, A.S. Sergeev, V.Yu. Zaslavsky, I.V. Zheleznov, Highpower terahertz-range planar gyrotrons with transverse energy extraction, Phys. Rev. Letters, v. 108, 105101 (2012)

2. V.Yu. Zaslavsky, N.S. Ginzburg, M.Yu. Glyavin, I.V. Zheleznov, I.V. Zotova, Threedimensional particle-in-cell modeling of terahertz gyrotrons with cylindrical and planar configurations of the interaction space, Phys. Plasmas, v. 20, 043103 (2013)

3. D. I. Sobolev, G. G. Denisov, Principles of synthesis of multimode waveguide units, IEEE Trans. Plasma Sci., v. 38(10), pp. 2825-2830 (2010) 